\title{
Differentiating between Patients with Alzheimer's Disease and Patients with Major Depressive Disorder Using the Voxel-based Specific Regional Aanalysis System for Alzheimer's Disease
}

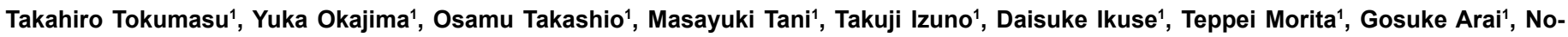
buyuki Saga1, Koji Hori2*, Takehiko Gokan ${ }^{1}$, Hiroshi Matsuda1 and Akira Iwanami ${ }^{1}$

${ }^{1}$ Department of Psychiatry, Showa University, Karasuyama Hospital, Kitakarasuyama, Setagayaku, Tokyo, Japan

${ }^{2}$ Department of Psychiatry, Showa University Northern Yokohama Hospital, 35-1 Chigasakichuo, Tsuzukiku, Yokohama City, Kanagawa, Japan

\begin{abstract}
Background: Recently, voxel-based morphometry (VBM) has become a popular tool for the early diagnosis of Alzheimer's disease (AD). The voxel-based specific regional analysis system for Alzheimer's disease (VSRAD) is a clinically useful VBM technique that employs magnetic resonance imaging (MRI) to automatically detect the loss of Powered by Editorial Manager $\AA$ and ProduXion Manager $\AA$ from Aries Systems Corporation gray matter volume in the medial temporal lobe.
\end{abstract}

Objective: To investigate the utility of VSRAD for differentiating between AD and major depressive disorder (MDD), and to identify the neuropathological differences between the two groups.

Methods: The subjects included 18 patients with MDD (mean \pm standard deviation: $74.8 \pm 7.1$ years, 4 males and 14 females) and 31 patients with $A D(82.4 \pm 7.3$ years, 7 males and 24 females). Three-dimensional T1-weighted sagittal images, were acquired using a 1.5Tesla MRI device and analyzed using the VSRAD advance software, parahippocampal atrophy was represented as a Z-score. Neuropsychological tests consisted of the Patient Health Questionnaire 9, Hamilton Rating Scale for Depression, Global Assessment of Function and Mini-Mental State Examination (MMSE). Correlations between the Z-score and the neuropsychological test scores were statistically examined.

Results: Patients with AD had significantly higher Z-scores than did patients with MDD (1.99 \pm 1.27 vs. $1.11 \pm 0.49$ $p<0.001$ ), and subjects with Z-scores > 2 were all diagnosed as AD. In the AD group, the Z-scores were significantly correlated with the MMSE scores throughout the study period ( 0 weeks: $p=0.015,24$ weeks: $p=0.024)$, whereas no significant correlations between the Z-scores and MMSE were observed for the MDD group. Conclusion: Our results obtained using the VSRAD suggest that VSRAD is useful for differentiating between AD and MDD, which is important, as the these two diseases are often difficult to diagnose based solely on their symptoms. Such findings imply that VSRAD may become a useful auxiliary diagnostic tool.

Keywords: Alziemer's disease; AD; MDD

\section{Introduction}

The age of Japan's population is advancing, leading to an increase in the number of older individuals who are at risk of developing dementia and late-life depression. One fourth of the Japanese population is over 65 years old, and Alzheimer's disease (AD) remains the most common cause of dementia among all age groups, while depression occasionally appears at the early stage of dementia in older individuals $[1,2]$. On the other hand, 30,000 people commit suicide every year and one third of them are over 60 years of age. In older individuals, major depressive disorder (MDD) is frequently associated with cognitive impairment and dementia-like symptoms [3,4]. Moreover, a previous study reported that both patients with dementia and patients with mild cognitive impairment are approximately 2.5 times more likely to have depression compared to healthy individuals [5]. However, a common clinical problem is distinguishing between $\mathrm{AD}$ and MDD, which is critical in order to adequately treat the patients. AD and MDD are sometimes difficult to diagnose at the first examination because MDD can progress into $\mathrm{AD}$, although this is not always the case. Therefore, a diagnostic tool that is useful for determining the longitudinal prognosis is required. Indeed, several reports have suggested that MDD might be a risk factor in $\mathrm{AD}$ [6]. Moreover, some forms of MDD are considered to constitute the prodromal symptoms of dementia [7]. Consistent with this notion, some reports have indicated that a history of depression is associated with an increased risk of developing $\mathrm{AD}$ [8]. Clearly, the association between MDD and $\mathrm{AD}$ is complicated, which makes diagnosis difficult. Regardless, it is important to distinguish between $\mathrm{AD}$ and MMD clinically and to determine which disorder is the major cause of the problem. Recently, voxel-based morphometry (VBM) has become a popular tool for the early diagnosis of $\mathrm{AD}$. One new free software program that has been gaining attention is the voxel-based specific regional analysis system for Alzheimer's disease (VSRAD), which is a VBM method that employs magnetic resonance imaging (MRI) to automatically detect the loss of gray matter volume in the medial temporal lobe, wherein the degree of atrophy is represented as a Z-score. VSRAD is designed to evaluate the relative local brain volume of individual patients by comparing the visual information from MR images with the brain image database for healthy individuals. According to VSRAD, individuals with a Z-score $>2$ are diagnosed with

*Corresponding author: Koji Hori, Department of Psychiatry, Showa University, Northern Yokohama Hospital 35-1, Chigasakichuo, Tsuzukiku, Yokohama City Kanagawa-224-8503, Japan, Tel: +81459497000, Fax: +81459497927; E-mail kojihori@med.showa-u.ac.jp

Received October 26, 2015; Accepted November 19, 2015; Published November 25, 2015

Citation: Tokumasu T, Okajima Y, Takashio O, Tani M, Izuno T, et al. (2015) Differentiating between Patients with Alzheimer's Disease and Patients with Major Depressive Disorder Using the Voxel-based Specific Regional Aanalysis System for Alzheimer's Disease. Brain Disord Ther 4:196. doi:10.4172/2168-975X.1000196

Copyright: (c) 2015 Tokumasu T, et al. This is an open-access article distributed under the terms of the Creative Commons Attribution License, which permits unrestricted use, distribution, and reproduction in any medium, provided the original author and source are credited. 
Citation: Tokumasu T, Okajima Y, Takashio O, Tani M, Izuno T, et al. (2015) Differentiating between Patients with Alzheimer's Disease and Patients with Major Depressive Disorder Using the Voxel-based Specific Regional Aanalysis System for Alzheimer's Disease. Brain Disord Ther 4:196. doi:10.4172/2168-975X.1000196

Page 2 of 5
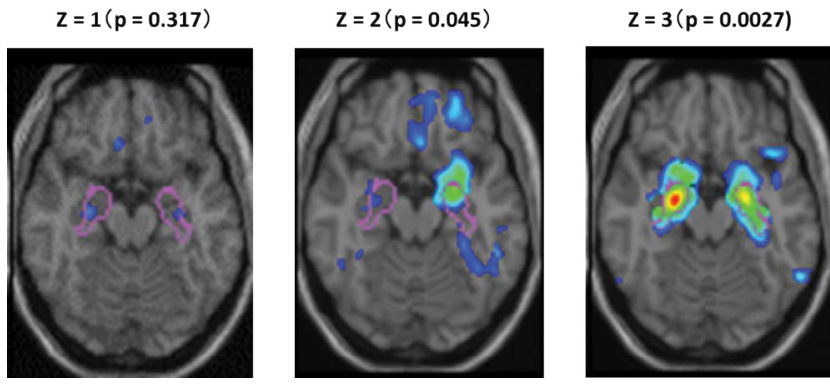

Figure 1: Z-score and corresponding parahippocampal atrophy samples with its statistical significance.

$\mathrm{AD}$ at 0.045 probability level (Figure 1$)$. Correlations between atrophy of the entorhinal cortex and cognitive function are well known. It is also reported that atrophy of the entorhinal cortex reflects not only the earliest pathological changes of $\mathrm{AD}$ to help early diagnosis, but also the course of progression to follow up the disease [9]. However, no study have researched VSRAD as a diagnostic tool for differentiating between $\mathrm{AD}$ and $\mathrm{MDD}$ and followed them prospectively.

The purpose of this study was to apply VSRAD to patients with possible $\mathrm{AD}$ and patients with possible MDD in order to distinguish between the two diseases. We also investigated the validity of VSRAD for cross-sectional and longitudinal diagnoses, which is important for establishing treatment strategies for the patients. Assuming that VSRAD would be useful for identifying the differences in parahippocampal atrophy between $\mathrm{AD}$ and $\mathrm{MDD}$, we generated three hypotheses and designed a long-term prospective study. Our first hypothesis was that patients with Z-scores $>2$ would be diagnosed with AD. Secondly, we hypothesized that patients with MDD would have lower Z-scores and a better prognosis compared to patients with $\mathrm{AD}$. Finally, we hypothesized that there would be a correlation between the Z-score and the Mini-Mental State Examination (MMSE) score in patients with $\mathrm{AD}$, while no such correlation would be observed in patients with MDD.

\section{Methods}

\section{Subjects}

Participants recruited for this study were outpatients of Showa University Hospital and Showa University Karasuyama Hospital who were over 60 years of age and who met the Diagnostic and Statistical Manual of Mental Disorders, Fourth Edition, Text Revision (DSMIV-TR) criteria for AD or MDD. Participants were excluded from this study if they met the criteria for another major psychiatric illness (schizophrenia, schizoaffective disorder, bipolar disorder) or had neurological illnesses. Patients who had received electroconvulsive therapy and those with a history of brain injury were also excluded from the study. Forty-nine subjects with a mean age of 79.5 years (range: 62-97 years) were eventually enrolled. Of the 49 subjects, 11 were male and 38 were female. The average duration of disease was 49.5 months (range: 1-276 month[s]). Participants were divided into two groups, the AD group and the MDD group, according to the DSM-IV-TR criteria. Details of the participant demographics are listed in Table 1.

\section{Assessment}

MRI scans for the VSRAD analysis were obtained between December 2013 and December 2014. The image was acquired only once at the initial interview (week 0). General cognitive function was assessed with the MMSE. The symptoms and severity of depression were assessed using the 17-item Hamilton Rating Scale for Depression (HRSD), and the Patient Health Questionnaire 9 (PHQ9) was used as a self-rated measurement of depressive symptoms. The Global Assessment of Function (GAF) test was also administered at the initial interview. These neuropsychological tests were also assessed at 8 weeks and 24 weeks after the MRI scan.

\section{MRI procedure and VSRAD analysis}

MRI was performed using a GE Signa 1.5-Tesla scanner (GE Signa, Milwaukee, WI) with the following parameters: flip angle, $25^{\circ}$; repetition time, $8.5 \mathrm{~ms}$; echo time, $3.7 \mathrm{~ms}$; field of view, $23 \times 23 \mathrm{~cm}$; matrix size, $256 \times 256$; and 1.4-mm slice thickness. Data obtained from the MR images were automatically analyzed using the VSRAD advance software (Eisai Co., Ltd., Tokyo, Japan). First, equalization of the voxel sizes and linear and nonlinear transformations were performed. Then, images of the gray matter, white matter, and cerebrospinal fluid were separated, and the gray matter images were standardized and smoothed onto templates using DARTEL. The gray matter image of a patient was compared with the mean and standard deviation of the gray matter images of the healthy volunteers using a voxel-by-voxel Z-score analysis, as follows: Z-score $=([$ control mean] - [individual value] $) /$ (controlstandard deviation) [10]. Thus, the Z-score represents the parahippocampal atrophy compared to healthy controls, wherein a

\begin{tabular}{|c|c|c|c|c|}
\hline & Total $(n=49)$ & $A D^{*}(n=31)$ & $M D^{*}(n=18)$ & P-value \\
\hline \multicolumn{5}{|c|}{ Sex, $\mathbf{n}$} \\
\hline Male & 11 & 7 & 4 & NS \\
\hline Female & 38 & 24 & 14 & \\
\hline Age, years & $79.5 \pm 8.0$ & $82.1 \pm 7.4$ & $75.0 \pm 7.2$ & $<0.005$ \\
\hline Disease duration, Month & $49.5 \pm 64.0$ & $51.6 \pm 68.6$ & $46.0 \pm 56.9$ & NS \\
\hline Z-score, $n$ & $1.65 \pm 1.12$ & $1.99 \pm 1.23$ & $1.07 \pm 0.51$ & $<0.005$ \\
\hline \multirow{2}{*}{$\geq 2$} & 10 & 10 & 0 & \multirow{2}{*}{$<0.01$} \\
\hline & $<10>^{* *}$ & $<$ AD10 MDD0 $>* *$ & & \\
\hline \multirow{2}{*}{$<2$} & 39 & 21 & 18 & \\
\hline & $<39>^{* *}$ & $<$ AD19 DD2>** & $<$ AD1DD17 $\rangle^{* *}$ & \\
\hline
\end{tabular}

Notes. Demographic information for the subject. Values are mean \pm standard deviation unless otherwise specified. NS=not significant at an alpha of 0.05. AD: Alzheimer's Disease; MDD: Major Depressive Disorder. * : initial diagnosis; <>** final diagnosis.

Table 1: Participant demographics

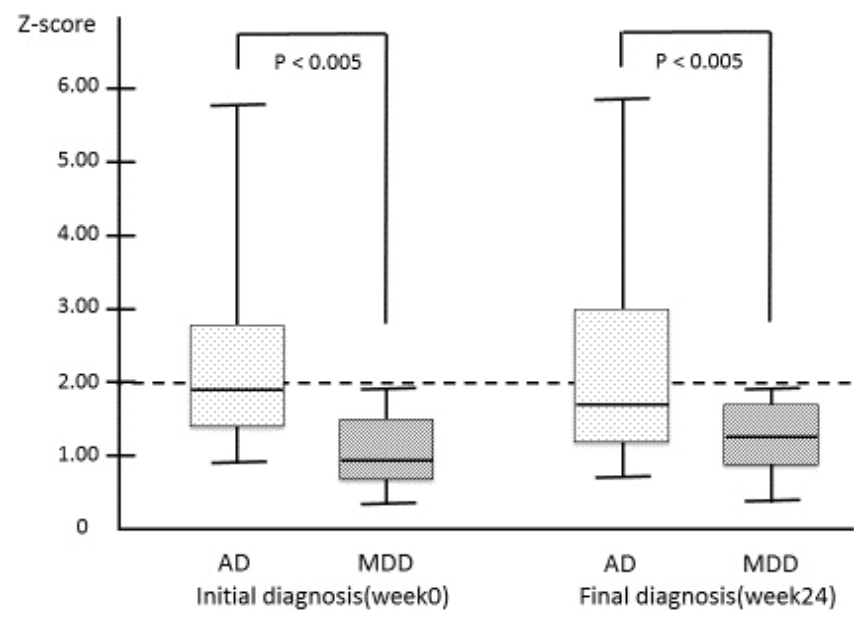

Figure 2: Comparison of Z-score between the AD and MDD groups. 
Citation: Tokumasu T, Okajima Y, Takashio O, Tani M, Izuno T, et al. (2015) Differentiating between Patients with Alzheimer's Disease and Patients with Major Depressive Disorder Using the Voxel-based Specific Regional Aanalysis System for Alzheimer's Disease. Brain Disord Ther 4:196. doi:10.4172/2168-975X.1000196

Page 3 of 5

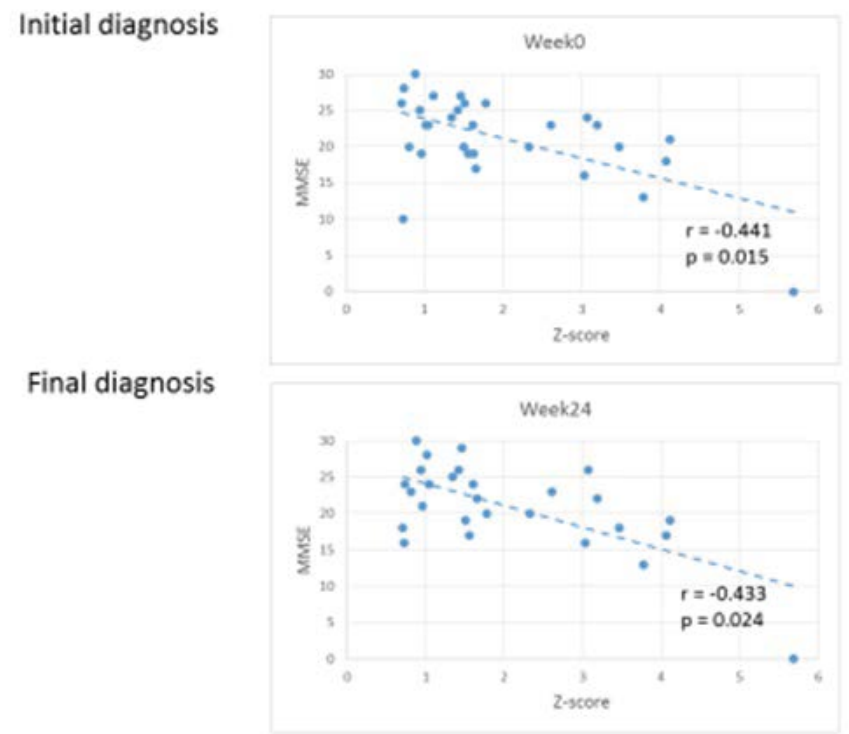

Figure 3: Correlation between the MMSE score and the Z-score in the AD group.

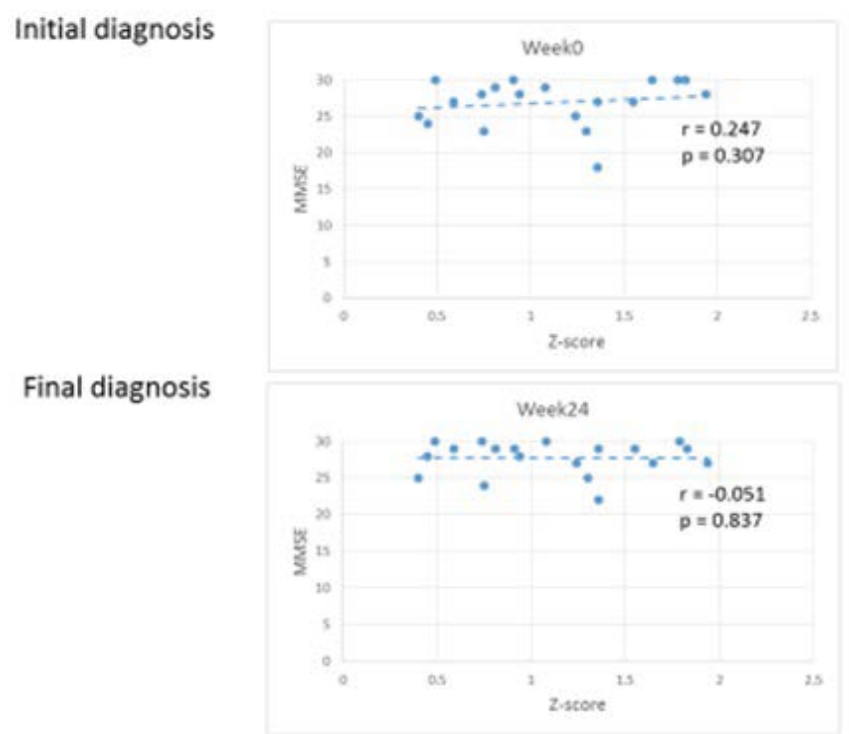

Figure 4: Correlation between the MMSE score and the Z-score in the MDD group.

Z-score of $<1$ meant no atrophy, $1<$ Z-score $<2$ meant mild atrophy, and a Z-score of $>2$ was defined as significant atrophy according to previous studies (Figure 1) [11]. The database for healthy individuals includes 40 Japanese males and 40 Japanese females between 54 and 86 years of age (70.2 \pm 7.3 years).

\section{Statistical analysis}

Analyses were performed using the Statistical Package for Social Sciences (SPSS) version 23 (SPSS, Inc., Chicago, IL, USA); a p-value of $<0.05$ was considered statistically significant. Data are reported as the mean \pm the standard deviation, unless otherwise specified. Basic group comparisons were performed by Mann-Whitney $U$ tests and the $\chi^{2}$ test for categorical variables. Spearman's correlation coefficient $(r)$ and partial correlation analyses were used to evaluate the correlations among the VSRAD Z-scores, demographic data, and neuropsychological test scores. Mixed-model analysis to repeated measures ANOVA were used to obtain the chronological change in the neurological test scores.

\section{Ethics}

This study was conducted with the approval of the ethics committee of Showa University Hospital and Showa University Karasuyama Hospital. The purpose of the study was explained to all subjects, both verbally and in written form, and their written informed consent was obtained.

\section{Results}

The AD group had significantly higher Z-scores than did the MDD group $(\mathrm{p}<0.001)$. Ten subjects had $\mathrm{Z}$-scores $>2$, and they were all diagnosed as having $\mathrm{AD}$; this diagnosis did not change after 24 weeks (Table 1). The Z-score was significantly higher in the AD group compared to the MDD group at the initial diagnosis (week 0) and at the final diagnosis (week 24). In the MDD group, all subjects had $\mathrm{Z}$-scores $<2$ (Figure 2 ). In the $\mathrm{AD}$ group, the $\mathrm{Z}$-scores were significantly negatively correlated with the MMSE score throughout the study period (Figure 3), whereas in the MDD group, no significant correlation was found (Figure 4). No correlation was identified between the Z-score and age in either group. One patient with MDD and two patients with

\begin{tabular}{|c|c|c|c|c|}
\hline C & & Week 0 & Week 8 & Week 24 \\
\hline \multirow{2}{*}{ MMSE } & $<\mathrm{AD}\rangle^{* *}$ & $22.1 \pm 4.8$ & $21.8 \pm 5.1$ & $22.4 \pm 4.5$ \\
\hline & $<M D D>^{* *}$ & $26.7 \pm 3.3$ & $27.5 \pm 2.0$ & $27.9 \pm 1.9$ \\
\hline \multirow{2}{*}{ HRSD } & $<\mathrm{AD}>^{* *}$ & $5.1 \pm 3.6$ & $3.5 \pm 3.3$ & $4.7 \pm 5.6$ \\
\hline & $<M D D>^{* *}$ & $9.6 \pm 5.6$ & $5.9 \pm 4.9$ & $5.9 \pm 4.0$ \\
\hline \multirow{2}{*}{ PHQ9 } & $<A D>^{* *}$ & $4.7 \pm 3.8$ & $4.1 \pm 3.2$ & $3.1 \pm 2.5$ \\
\hline & $<M D D>^{* *}$ & $7.6 \pm 7.3$ & $5.3 \pm 4.5$ & $6.6 \pm 6.0$ \\
\hline \multirow{2}{*}{ GAF } & $<\mathrm{AD}>^{* *}$ & $58.4 \pm 13.5$ & $68.1 \pm 18.0$ & $62.4 \pm 18.6$ \\
\hline & $<$ MDD $>^{* *}$ & $66.6 \pm 9.1$ & $69.2 \pm 10.6$ & $70.0 \pm 9.8$ \\
\hline
\end{tabular}

AD: Alzheimer's disease; MDD: Major depressive disorder; MMSE: The MiniMental State Examination; HRSD: The Hamilton Rating Scale for Depression; PHQ9: The Patient Health Questionnaire-9; GAF: The modified Global Assessment of Functioning.

Table 2: Chronological changes in the neurological tests (grouped by final diagnosis).

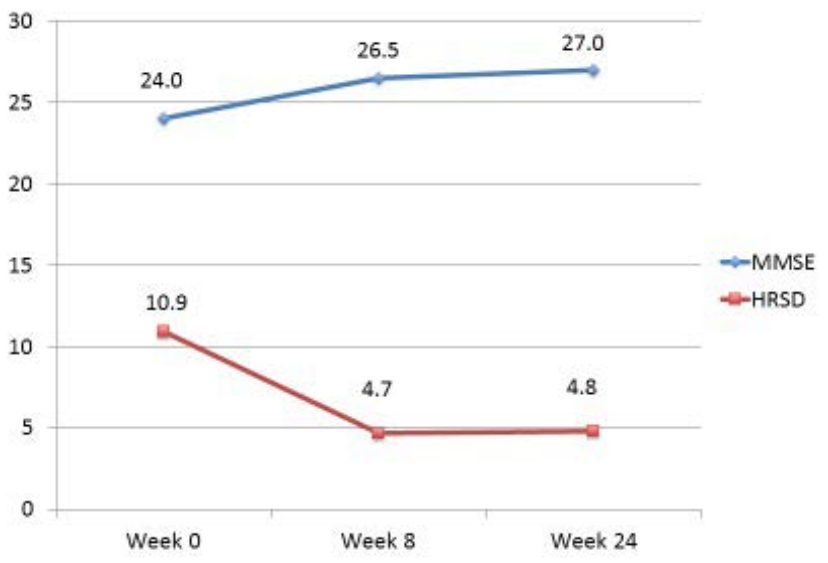

zescore

Figure 5: Chronological changes in the MMSE and HRSD scores in patients with MDD with MMSE below 27. 
Citation: Tokumasu T, Okajima Y, Takashio O, Tani M, Izuno T, et al. (2015) Differentiating between Patients with Alzheimer's Disease and Patients with Major Depressive Disorder Using the Voxel-based Specific Regional Aanalysis System for Alzheimer's Disease. Brain Disord Ther 4:196. doi:10.4172/2168-975X.1000196

$\mathrm{AD}$ had a different diagnosis at 24 weeks. A partial correlation analysis was performed for age, but the results did not change. The results of the mixed model analysis of variance revealed significant differences in all four neurological tests. The MMSE scores were significantly higher in the $\mathrm{AD}$ group $(\mathrm{p}<0.001)$, although chronological change and interaction effects were not found. Improvement in the HRSD score was obvious from week 0 to week 8 , especially in the MDD group $(p<0.005)$. No significant difference in HRSD was found between the two groups $(\mathrm{p}=0.15)$. The PHQ9 score significantly improved from week 0 to week $8(\mathrm{p}<0.05)$, but there was no difference between the two groups and no interaction effect was found. The GAF score was significantly lower in the AD group $(\mathrm{p}<0.05)$ and both groups showed chronological improvement $(\mathrm{p}<0.01)$, although no interaction effect was found (Table 2). When we focused on patients with MMSE scores below 27 in the MDD group, we found significant improvement in the MMSE score from week 0 to week $24(24.00 \pm 2.89$ vs. $27.00 \pm 2.07$, $\mathrm{p}=0.022)$. We also observed a significant decrease in the HRSD score in the MDD group at week $8(\mathrm{p}=0.01)$ and at week $24(\mathrm{p}=0.004)$ (Figure 5).

\section{Discussion}

To our knowledge, this is the first study to establish the validity of using VSRAD to discriminate patients with $\mathrm{AD}$ from patients with MDD and the first to observe the changes on a long-term basis. Our results showed that subjects with Z-scores $>2$ were all diagnosed as having $\mathrm{AD}$, and these diagnoses did not change after 24 weeks. Moreover, we found that the MDD group had significantly lower Z-scores than did the AD group; in particular, we found significant improvement in the cognitive symptoms and depressive symptoms of MDD patients with MMSE scores below 27. There was also a strong negative correlation between the Z-scores and the MMSE scores in the $\mathrm{AD}$ group throughout our study period; however, this correlation was not found in the MDD group. Collectively, these findings indicated that VSRAD is useful for differentiating between AD and MDD with regards to both the cross-sectional and longitudinal diagnoses, which is important for establishing treatment strategies for these disorders. Many previous studies have reported that Z-scores $>2$ indicate a probable diagnosis of $\mathrm{AD}$; however, this is the first study to investigate the $\mathrm{Z}$-scores on a long-term basis. In this study, no improvement in the MMSE scores was observed in patients with AD. A Z-score $>2$ with cognitive impairment strongly indicates the existence of $\mathrm{AD}$ and suggests an early $\mathrm{AD}$ treatment regardless of their depressive symptoms. Meanwhile, a Z-score $<2$ might indicate the need to consider the coexistence of $A D$ and MDD. In the present study, the MDD group had significantly lower Z-scores than did the AD group, and in some MDD patients, improvement in the cognitive symptoms was observed. This indicates that in the AD group, the MMSE score decreased as a result of parahippocampal atrophy, which was represented as higher Z-scores. In the MDD group, the MMSE score decreased as a sequel to depressive symptoms and unrelated with parahippocampal atrophy. However, even though the HRSD scores improved in the MDD group at week 8 and week 24 compared to the scores at week 0, the MMSE scores did not significantly improve. This was inconsistent with our theory because we expected that in MDD group, MMSE would improve as they recover from depressive symptoms. This was due to the fact that there were several MDD patients with full MMSE scores (30 points) from week 0 , which might have diminished the statistical difference in the chronological MMSE changes. Accordingly, we focused on patients with MDD whose MMSE scores were below 27 and found significant improvement in the MMSE score from week 0 to week 24 . In the AD group, the Z-scores were strongly negatively correlated with the MMSE score, whereas no such correlation was found in the MDD group. This indicates that there are neuropathological differences between $\mathrm{AD}$ and MDD that do not change for at least 24 weeks. However, contrary to our hypothesis, we did not identify a relationship between the Z-scores and the chronological changes in the MMSE scores in the AD group. This could be due to the anti-dementia drug treatment of AD during our study period, and the 24-week study period may have been too short to observe changes in the MMSE score. This indicates that patients with MDD with Z-scores $<2$ are likely to show improvements in their depressive symptoms.

Structural MRI studies and functional studies using fluorodeoxyglucose-positron emission tomography and single photon emission computed tomography are widely used in the diagnosis of AD. The results of a previous single photon emission computed tomography study suggest that there is a correlation between the VSRAD Z-scores and regional cerebral blood flow in patients with $\mathrm{AD}$ [12]. Near infrared spectroscopy is also reportedly a useful tool in the differential diagnosis of $\mathrm{AD}$ and MDD [13]. However, none of these methods is known to be satisfactory from a perspective of versatility and diagnostic accuracy. The new VBM-based VSRAD advance software automatically analyzes MRI data and is able to overcome the shortcomings of region-of-interest analyses; moreover, it is reportedly more accurate than manually defined region-of-interest-based analyses at discriminating patients with mild to moderate $\mathrm{AD}$ from controls [14]. Traditional VSRAD studies indicate that there are positive correlations between the Z-score and cognitive symptoms, which is consistent with findings of the present study. Previous research also suggests that the parahippocampal atrophy is a particularly useful index for discriminating patients with mild $\mathrm{AD}$ from healthy controls and is a sensitive predictor of conversion to AD [15]. It should be noted that previous VBM studies indicate that there is a correlation between AD and late-life depression [16,17]. Recent VSRAD studies indicate that unlike $\mathrm{AD}$, patients with late-life depression show no evidence of atrophy in the precuneus and posterior cingulate cortex [18]. These findings suggest that changes in the more posterior parts of the brain may be more likely to be observed in AD. Moreover, patients with mood disorders show atrophy in the subgenual anterior cingulate cortex and subcallosal anterior cingulate cortex [19]. Here, we observed almost no changes in the patients' diagnoses 24 weeks after the initial interviews. These results imply that the skill of the psychiatrist during the diagnostic interview is the most important, while VSRAD is useful but remains a supplemental tool.

Our study had several limitations. First, diagnosis could change after 24 weeks and more MDD patients may develop AD over time. Second, the findings reported in the study should be interpreted with caution because of the small sample size. Third, a few patients did not complete the neuropsychological tests, which may have caused deviations in our research. Fourth, the two groups were not matched with regards to age, disease severity, disease duration, treatment period, type of drug used, or drug dose. Age was also not matched with healthy control, which may have affected the results. Finally, patients only underwent 1.5 -Tesla MRI scanning at the initial phase; a second MRI scan at 24 weeks and the determination of the volume of CA1 and subiculum, and cortical signatures through 3-Tesla MRI would have provided us with more information about the progression of $\mathrm{AD}$ and the $\mathrm{Z}$-scores. Further studies are required to investigate these issues.

\section{Conclusion}

The present study suggests that VSRAD is useful for discriminating $\mathrm{MDD}$, a disorder with symptoms that are difficult to diagnose, from $\mathrm{AD}$, 
Citation: Tokumasu T, Okajima Y, Takashio O, Tani M, Izuno T, et al. (2015) Differentiating between Patients with Alzheimer's Disease and Patients with Major Depressive Disorder Using the Voxel-based Specific Regional Aanalysis System for Alzheimer's Disease. Brain Disord Ther 4:196. doi:10.4172/2168-975X.1000196

Page 5 of 5

using the Z-score $>2$ threshold due to differences in Para-hippocampal atrophy between the two patient groups. Although advances in the VBM technique have been made and the VSRAD software is routinely applied in clinical studies on AD in Japan, more studies are needed to clarify the validity of VSRAD. The findings provided herein suggest that VSRAD may become a useful auxiliary tool for diagnosing and monitoring the progression of $\mathrm{AD}$ worldwide.

\section{Acknowledgment}

The authors have no competing interests to declare.

\section{References}

1. Matsuda $\mathrm{H}$ (2007) The role of neuroimaging in mild cognitive impairment Neuropathology 27: 570-577.

2. Mizukami K (2013) Alzheimer's disease and depression. Psychiatria et Neurologia Japonica 115: 1122-1126.

3. Byers AL, Yaffe K (2011) Depression and risk of developing dementia. Nature Reviews. Neurology 7: 323-331.

4. Weisenbach SL, Boore LA, Kales HC (2012) Depression and cognitive impairment in older adults. Current Psychiatry Reports 14: 280-288.

5. Snowden MB, Atkins DC, Steinman LE, Bell JF, Bryant LL, et al. (2015) Longitudinal Association of Dementia and Depression. The American Journal of Geriatric Psychiatry 23(9): 897-905.

6. Diniz BS, Butters MA, Albert SM, Dew MA, Reynolds CF 3rd (2013) Late-life depression and risk of vascular dementia and Alzheimer's disease: systematic review and meta-analysis of community-based cohort studies. The British Journal of Psychiatry 202(5): 329-335.

7. Schweitzer I, Tuckwell V, O'Brien J, Ames D (2002) Is late onset depression a prodrome to dementia? Geriatric Psychiatry 17: 997-1005.

8. Ownby RL, Crocco E, Acevedo A, John V, Loewenstein D (2006) Depression and Risk for Alzheimer Disease Systematic Review, Meta-analysis, and Metaregression Analysis. Arch Gen Psychiatry 63: 530-538.

9. Tokuchi R, Hishikawa N, Kuruta T, Sato K, Kono S, et al. (2014) Clinical and demographic predictors of mild cognitive impairment for converting to Alzheimer's disease and reverting to normal cognition. Journal of the
Neurological Sciences 346: 288-292.

10. Matsuda H (2013) Voxel-based Morphometry of Brain MRI in Normal Aging and Alzheimer's Disease. Aging and Disease 4(1): 29-37.

11. Hirata Y, Matsuda H, Nemoto K, Ohnishi T, Hirao K, et al. (2005) Voxel-based morphometry to discriminate early Alzheimer's disease from controls. Neurosci Lett 382: 269-274

12. Li X, Shimizu S, Jibiki I, Watanabe K, Kubota T (2010) Correlations between Z-scores of VSRAD and regional cerebral blood flow of SPECT in patients with Alzheimer's disease and mild cognitive impairment. Psychiatry and Clinical Neuroscience 64: 284-292.

13. Kito H, Ryokawa A, Kinoshita Y, Sasayama D, Sugiyama N, et al. (2014) Comparison of alterations in cerebral hemoglobin oxygenation in late life depression and Alzheimer's disease as assessed by near-infrared spectroscopy. Behavioral and Brain Functions 10: 8.

14. Testa C, Laakso MP, Sabattoli F, Rossi R, Beltramello A, et al. (2004) A comparison between the accuracy of voxel-based morphometry and hippocampal volumetry in Alzheimer's disease. Journal of Magnetic Resonance Imaging 19: 274-282.

15. Li X, Jiao J, Shimizu S, Jibiki I, Watanabe K, et al. (2012) Correlations between atrophy of the entorhinal cortex and cognitive function in patients with Alzheimer's disease and mild cognitive impairment. Psychiatry and Clinical Neurosciences 66: 587-593.

16. Palmer K, Di Lulio F, Varsi AE, Gianni W, Sancessario G, et al. (2010) Neuropsychiatric predictors of progression from amnestic-mild cognitive impairment to Alzheimer's disease: the role of depression and apathy. Journal of Alzheimer's Disease 20: 175-183.

17. Houde M, Bergman $\mathrm{H}$, Whitehead V, Chertkow $\mathrm{H}$ (2008) A predictive depression pattern in mild cognitive impairment. Geriatric psychiatry 23(10): 1028-1033.

18. Shimoda K, Kimura M, Yokota M, Okubo Y (2015) Comparison of regional gray matter volume abnormalities in Alzheimer's disease and late life depression with hippocampal atrophy using VSRAD analysis: A voxel-based morphometry study. Neuroimaging 232: 71-75.

19. Niida A, Niida R, Matsuda H, Motomura M, Uechi A (2014) Analysis of the presence or absence of atrophy of the subgenual and subcallosal cingulate cortices using voxel-based morphometry on MRI is useful to select prescriptions for patients with depressive symptoms. Int J Gen Med 7: 513-524. 